\title{
A Spearman Algorithm Based Brain Tumor Detection Using CNN Classifier forMRI
}

\section{Images}

\author{
Rachana Ramachandran.R.P, N.Mohanapriya, V.Banupriya
}

\begin{abstract}
Medical imaging and its processing is an area of interest which is helps for easier and analysis of the medical issues. These modalities can provide visual representations of the interior of a body for clinical analysis and medical interventions. Medical imaging also establishes a database of normal anatomy and physiology to make it possible to identify abnormalities. So it helping easier diagnosis and planning treatment. These detailed and informative mapping can be processed to exact the information instead of dealing with the whole data. The medical imaging technique plays a central role for diagnosis of brain tumors. During the recent years, the mortality rate of individuals due to brain tumor is rising rapidly. Brain tumor is a serious lifethreatening issue. Near the beginning and exact detection of brain tumor helps to reduces the brain tumor mortality rate, but it is a complicated and challenging task. To solve these difficulties use different brain tumor detection algorithms. Nowadays a number of brain tumor detection and classification algorithms are existing, but several classification processes have need of large time for classify the result. In order to improve the efficiency of brain tumor detection process, propose a spearman based brain tumor segmentation and Convolution Neural Network (CNN) based classification technique. This classifier provides best and accurate result. The proposed technique is estimated on the basis of their performance parameters on MRI brain images.
\end{abstract}

Keywords: Medical image processing, Brain tumor, CNN, Spearman algorithm, Segmentation, Classification, MRI

\section{INTRODUCTION}

Mind tumor is a party of headway of sporadic cells in the cerebrum. Cerebrum tumor might be malignant growth causing (unsafe) or noncancerous (kind). One of the most ridiculous unsafe issue is mind tumor. The end rate in light of mind tumor can be decreased by the use of certifiable and close starting disclosure, at any rate this is a badly designed assignment. Mind tumor treatment relies on different segments, for example, authentic confirmation and different parts like the tumor type, size, area and the state at which the tumor is made. In the past time of tumor perceived physically with the assistance of watching pictures by expert, to a great extent it requires additional time and results might be stirred up. Before long different PC based

Revised Manuscript Received on August 14, 2019.

Rachana Ramachandran.R.P, P.G Scholar, Dept.of Computer Science Engineering, Vivekanandha College of Engineering for Women, Tiruchengode, Namakkal, Tamilnadu, India. (E-mail: rachana.ram7@gmail.com)

N.Mohanapriya, Assistant Professor, Dept. of Computer Science Engineering, Vivekanandha College of Engineering for Women, Tiruchengode, Namakkal, Tamilnadu, India. (E-mail: mohanapriya@vcew.ac.in)

V.Banupriya, Associate Professor, Dept. Of Computer Science and Engineering, Vivekanandha College of Engineering for Women, Tiruchengode, Namakkal, Tamilnadu, India. (E-mail: malathi@vcew.ac.in) instrument is utilized in therapeutic field. These gadgets give energetic and unequivocal outcome.

Remedial picture preparing is an evaluation area that has conspicuously made in late decades. It has massive application district for expelled clinical issues. Restorative imaging is utilized to disclose inside structures concealed by the skin and bones, to dismember and treat sickness. Before long one of the snappiest making spaces for accommodating improvement is remedial imaging. Late remedial imaging structures are fit for give enormous extent of pictures, which needs all around assessment. X-bar (Magnetic Resonance Imaging), X-Ray, CT (Computed Tomography), PET (Positron Emission Tomography) and Ultrasound, and so on are ordinarily utilized helpful imaging improvements for picturize human organs.

Recognizing evidence of mind tumor utilizing picture arranging structure contains various stages, picture predealing with, division, highlight extraction and social event. The pre-preparing system is utilized for improve mind pictures by evacuating the unessential disturbance and undesired parts. In division the pre-masterminded cerebrum picture is changed over into parallel picture position. Highlight extraction is the methodology for social event higher level data of a picture. All together strategy, the classifier is utilized to bundle the information picture tests, to get best outcome.

These days diverse cerebrum tumor ID also as depiction structures are announced. In any case, just an obliged measure of highlights are separated in highlight extraction process, so the exactness is low by ethicalness of tumor recognizing confirmation. Two or three classifiers set aside longer effort to organize the outcome. To improve the capacity of gathering precision and abatement the unmistakable confirmation whimsy joins into the helpful picture managing predicted another strategy for mind tumor division and depiction. Spearman strategy based division approach and CNN based solicitation. In light of execution parameters on MR cerebrum pictures of proposed strategy have been studied regarding exactness, affectability and aura.

\section{EXISTING WORK}

Magnetic resonance images are the mainly well-known mode of detection and analysis of growth of tumors and also recognize its location. The affected cells are to be guessed by visualization process from MRI images is biased in to nature and have far above the ground probability of error

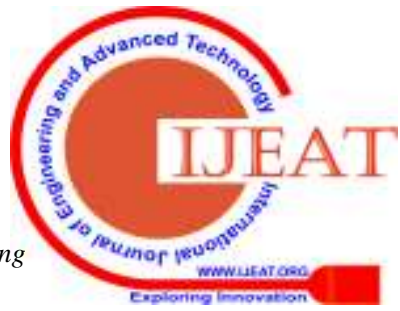




\section{A SPEARMAN ALGORITHM BASED BRAIN TUMOR DETECTION USING CNN CLASSIFIER FORMRI IMAGES}

happening process. Human machine communication system which helps the health proficient to detect tumor cells and increase the support of some machine learning algorithms. The dissimilarity in formation and size of cells certainly not interfere in the case of algorithm accuracy. Efficient segmentation of brain cells as gray matter, white matter for dimensionality reduction process and involved cancerous area can be accompanied [1]. But this method has very low accuracy for tumor detection.

Bandana Sharma and Brij Mohan Singh [2] focused on image processing for medical system using hybrid applications. This task mainly focused on the methods in image processing such as image acquisition, image preprocessing, segmentation, feature extraction, feature selection and classification. For each stage they introduced different algorithms.

Nikita V.Chavan et al [3] presented a method for identify and then classify the tumor as its primary stage. There are mainly two stages used for extracting the features of tumors and tumor classification. The first stage consists of using GLCM for collecting the MRI image characteristics. The final stage of classification of MRI brain is made by using K-nearest neighbour (KNN) classifier.

Anupurba Nandi [4] introduced a morphological operator based brain tumor detection technique. Thresholding and watershed method is used for segmentation. But it is difficult to determine and also this factor might be indisputable for various pictures. The consider utilized thresholding is exceptionally hard to pick and the watershed procedure is fundamentally delicate to close-by minima. Vrushali Borase et al [5] investigated MRI based character tumor zone approach utilizing fake neural structure. This system utilizes PC based methodologies to perceive tumor squares and depict the sorts of tumor utilizing Artificial Neural Network (ANN) mean MRI pictures of various patients. The basic disadvantage of this work is that the expenses are legitimately unpredictable to demonstrate the yield picture as a piece of the information pictures. Nicolas Coquery, Olivier Francois et al [6] inspected a more diminutive scale vascular MRI and solo assembling of histology looking like picture models. This procedure is utilized to make histology-looking like pictures dependent on tissue little scale vascularisation. This work contains six MRI parameters. Removing a MRI parameter from the assessment did not improve the general tissue depiction. Imaging complex mischief wounds, which are heterogeneous in nearness, is a genuine test.

Several methods can be used to represent the conventional stages of medical image processing and also analyze the individual segmentation approach [7]. Different methods are proposed to conclude the importance of machine learning in brain tumor classification and detection of brain tumors by using appropriate segmentation. Sometimes morphological operators can be used for feature selection [8]. Rohini and Ragan explored feature extraction based brain tumor detection. They also utilize some efficient classification method for detecting tumor areas in MRI brain images. Filters are used for removing noises in images. The final classification outputs become better and accurate. Due to the density and different types of tumors, the tumor detection process becomes too tough [9]. Machine learning algorithm based classification approaches are more efficient for MRI based brain tumor detection to get either normal or abnormal image output [11]. The feed-forward back-prop neural network based classification method is also available for brain tumor detection [10].

\section{PROPOSED WORK}

In the proposed method the given MRI brain input image is pre-processed by Gaussian filter. The partial identification of tumor region is done by median filter. The pre-processed images are fed in to segmentation module. In segmentation process, the pre-processed MRI brain image is converted into binary format. Discrete Wavelet Transform (DWT) is used for this purpose. Accurate recognition of tumor region is done by segmentation. The spearman algorithm is used to find the pixel size or magnitude of the samples for calculates the signals for tumor. After calculation the magnitude, then the signal is ready for classification. To enhance the segmented images, threshold based optimization technique is implemented with CNN classifier. Due to the presence of severe intensity variations, the proposed classifier has the ability to identify abnormal region present in the input image. Hence brain images with tumor are identified more appropriately.

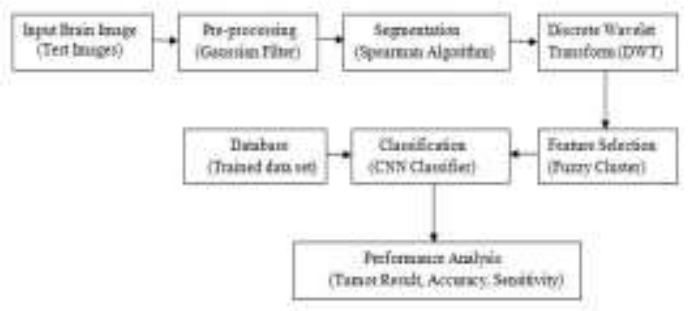

Figure 3.1 Block Diagram of Proposed System

\section{Module Description:}

\section{Pre-processing}

It consists of data pre-processing section. In this section, the given input data is ready for pre-processing. Preprocessing is used for removing irrelevant and undesired parts present in the input MRI brain image. Gaussian filer is used for this purpose. The Gaussian filter is a nonlinear method used to eject noise from input images, without reducing the sharpness. The Gaussian filter is a linear filter. It is generally used to blur the image or to decrease noise. In most of the signal processing system uses Gaussian filter. This filter have a property that, while minimizing the rise and fall time there is no overshoot to a step input function. Gaussian filter has different applications. The canny edge detector used in image processing uses Gaussian filter. And also it used in GFSK (Gaussian Frequency Shift Keying).

\section{Segmentation}

In segmentation, the pre-processed MRI brain image is converted into its binary format. In this process, the spearman algorithm is used to get the magnitude or pixel 
size of the sample from input image for calculates the signal for tumor by using the equation,

$$
\frac{1-6^{2}}{2-1}
$$

Where „n $\mathrm{n}^{\text {ee }}$ is the number of bits and „, $\mathrm{D}^{\mathrm{ee}}$ is the individual sample number. After calculating the magnitude, then the signal is ready for classification. For segmentation process, the DWT is used to convert the continuous signals into discrete samples.

\section{Feature Selection}

The feature selection is a process contained by a subset of features which can right to use from the selected data for learning calculation. This method is commonly used as element of machine learning. Altering the information on behalf of the arrangement of feature is called highlight extraction. The information to an estimation is irrationally huge for masterminded to be and it is relied on to be detestably monotonous then the information will be changed into a reduced portrayal set of highlights called as highlight vector. The part extraction is gainful for perceiving cerebrum tumor where certainly found and aides in imagining next stage. In highlight extraction is a system of get-together higher-level data of a picture such independent, centrality, entropy, homogeneity, affiliation, and so forth. One of the most typically utilized picture assessment structure is Gray Level Co-event Matrix (GLCM). The fragment extraction is achieved using GLCM. In feature selection process, some the extracted features are to be selected.

\section{Classification}

It consists of classifier evaluation unit. Here, CNN classifier is used to classify the trained and the test data set, from this get the best classifier output. To classify the input image of the proposed system, it required less time as compared with the existing system. A different layer of $\mathrm{CNN}$ is shown in figure 3.2 .

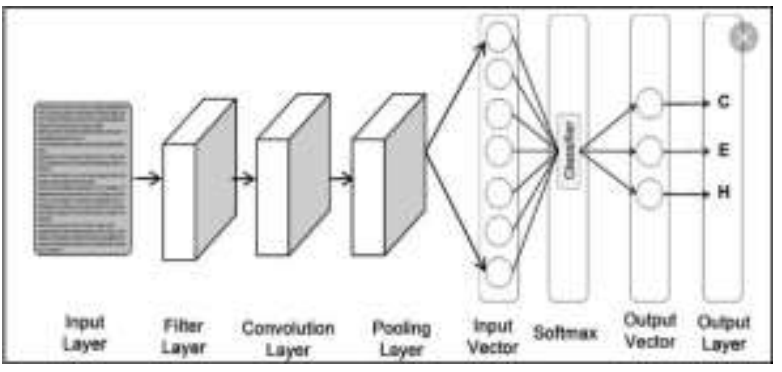

Figure 3.2 Layers used in CNN

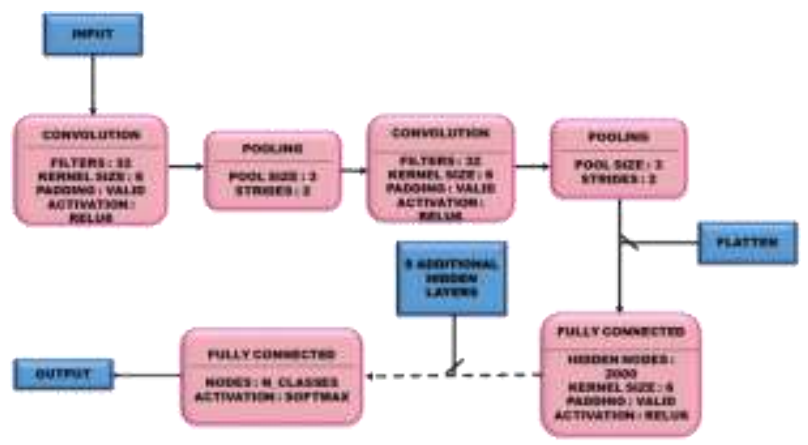

Figure 3.3 Proposed CNN Structure

\section{PERFORMANCE ANALYSIS}

Different parameters are used for evaluating the performance of algorithms. Accuracy, sensitivity and specificity are the parameters used to evaluate the performance of proposed system. CNN classifier gives best and accurate result. The performance of the proposed classification can be increased by increasing the number of iterations (Table 3.1).

\section{Table 3.1 Performance of CNN Classification}

\begin{tabular}{|c|c|c|c|}
\hline \multirow{2}{*}{ Parameters } & \multicolumn{3}{|c|}{$\begin{array}{l}\text { No of } \\
\text { Iterations }\end{array}$} \\
\cline { 2 - 4 } & 20 & 60 & 100 \\
\hline Accuracy (\%) & 26 & 34 & 42 \\
\hline Sensitivity (\%) & 27.5 & 29 & 32.5 \\
\hline Specificity (\%) & 30 & 32.5 & 36.5 \\
\hline
\end{tabular}

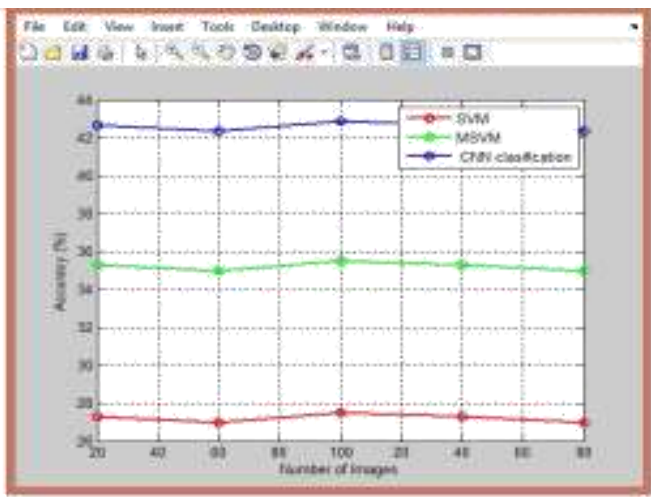

Figure 4.1 Accuracy of CNN Classifier

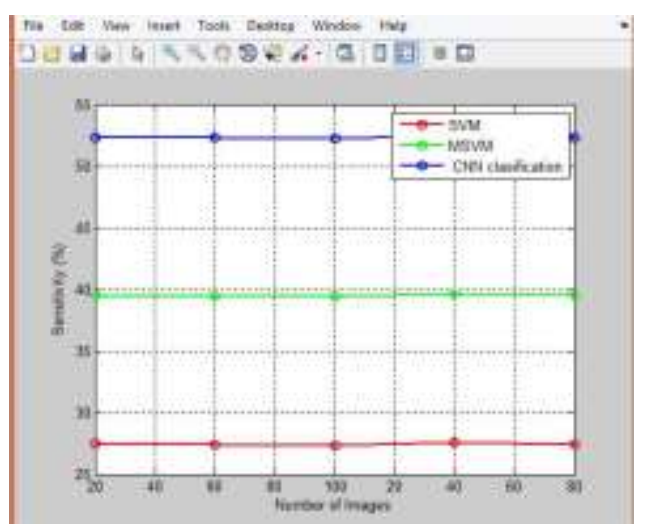

Figure 4.2 Sensitivity of CNN classifier 


\section{A SPEARMAN ALGORITHM BASED BRAIN TUMOR DETECTION USING CNN CLASSIFIER FORMRI IMAGES}

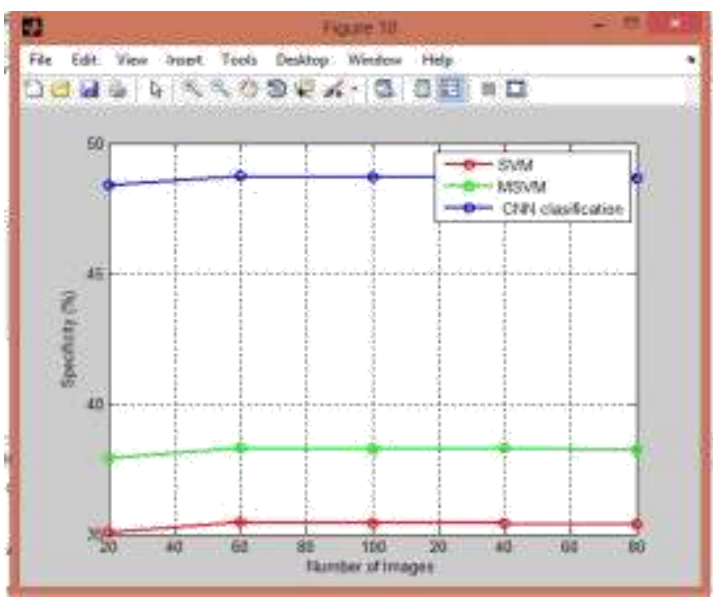

Figure 4.3 Specificity of CNN Classifier

\section{RESULTS AND DISCUSSION}

The results of MRI brain images for CNN classifier is given below for various input images. And the figures show that the output of MRI brain images for three different input images for CNN classifier. Figure 4.1, figure 4.2 and figure 4.3 shows tumor detected input images. The figure (a) denotes the input MRI brain image. (b) Denotes the preprocessed image using median filter. (c) Denotes the segmented MRI brain image and (d) denotes the edge detected MRI brain image. The results of proposed system show that it has better performance than existing system.
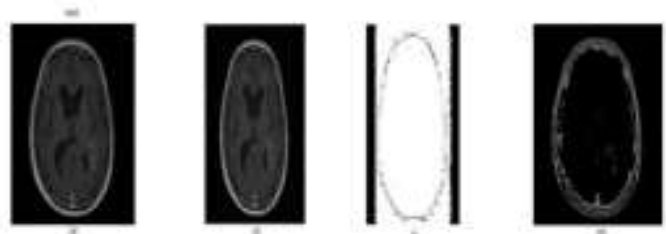

Figure 5.1 Tumor detected image 1
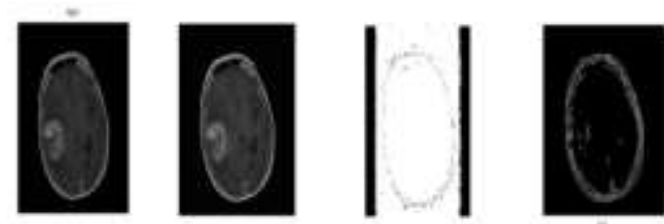

Figure 5.2 Tumor detected image 2
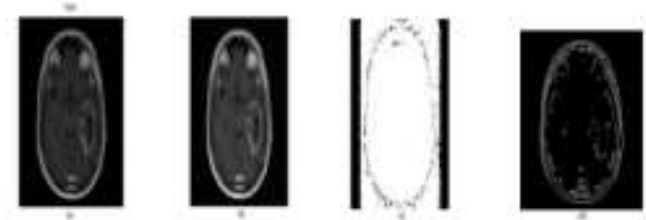

Figure 5.3 Tumor detected image 3

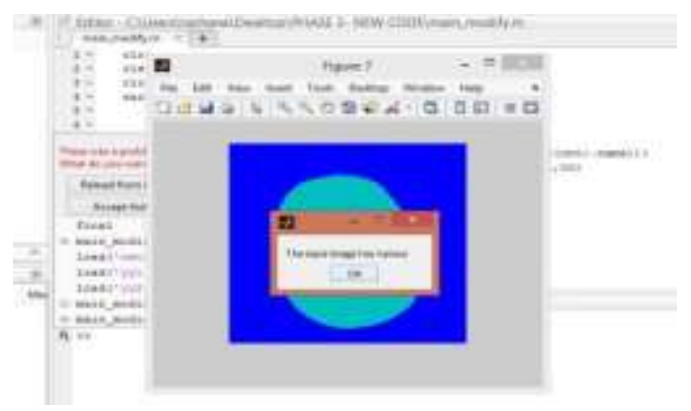

Figure 5.4 Tumor Result

\section{CONCLUSION \& FUTURE SCOPE}

One of the most serious life threatening issues is brain tumor. The image processing technique plays an important role for detecting brain tumors. The death rate due to brain tumor can be reduced by the help of near the beginning and precise brain tumor detection. But it is complicated and tough task. A number of brain tumor detection and classification algorithms are available, but some classification process required more time to classify the result. To improve the efficiency of classification and segmentation process, propose a spearman algorithm based segmentation and CNN based classification. The proposed classifier provided best and accurate result with less time duration. The main advantages of this proposed work is that, no need to have large trained dataset, low memory space is enough to maintain the dataset and the $\mathrm{CNN}$ based classification method provides more accurate result. Accuracy, sensitivity and specificity are the three parameters used to evaluate the proposed work. After the evaluation on performance parameters, this proposed method provides best result. In future, the proposed technique can also be applied for other organs like liver, lungs, breast etc with the help of other imaging modalities.

\section{REFERENCES}

1 Bahadure, N B Ray, A.K, and Thethi, H.P. 1017, „Image analysis for MRI based brain tumor detection and feature extraction using biologically inspired BWT and SVM"e, International journal in Biomedical Imaging.

2 Bandana Sharma, Dr. Brij Mohan Singh, 2016 „Review Paper on Brain Tumor Detection Using Pattern Recognition Techniques"e, International Journal of Recent Aspects, pp.151-156.

3 Nikita V.Chavan, B.d.Jadhav and P.M.Patil. „Article: Detection and Classification of Brain Tumorse ${ }^{\text {ee }}$, International Journal of Computer Applications 112(8):48-53, February 2015.

4 Anupurba Nandi, "Detection of human brain tumour using MRI image segmentation and morphological operators" IEEE International Conference on Computer Graphics, Vision and Information Security (CGVIS), 2015.

5 Vrushali Borase, Gayatri Naik. And Vaishali Londhe, "Brain MR Image Segmentation for Tumot Detection using Artificial Neural “ International Journal Of Engineering And Computer Science ISSN:2319-7242 Volume 6 Issue1, 2017. 
6 Nicolas Conquery, Oliver Francois et al., "Microvascular MRI and unsupervised clustering and unsupervised clustering yields histology-resembling images in two rat models of glioma " Journal of Cerebral Blood Flow \& Metabolism 34, 1354-1326, 2014.

7 Amruta Pramod Hebli, Sudha Gupta 2016, „Brain Tumor Detection Using Image Processing: A Survey" Proceeding of $65^{\text {th }}$ IRF International Conference, pp. 978-93. 